\title{
A first case report of rare synchronous double cancers: malignant cutaneous melanoma and gastrointestinal stromal tumor
}

\author{
Barbara Zegarska', Maciej Nowacki ${ }^{2,3}$, Katarzyna Pietkun ${ }^{1,4}$, Wojciech Jozwicki ${ }^{3,5}$, Tomasz Nowikiewicz ${ }^{2,3}$, \\ Noor Y. Al-Obaidi ${ }^{6}$, Samy L Habib ${ }^{6,7}$, Wojciech Zegarski ${ }^{2,3}$
}

\begin{abstract}
${ }^{1}$ Chair of Cosmetology and Aesthetic Dermatology, Ludwik Rydygier Collegium Medicum in Bydgoszcz, Nicolaus Copernicus University in Torun, Poland

${ }^{2}$ Chair and Department of Surgical Oncology, Ludwik Rydygier Collegium Medicum in Bydgoszcz, Nicolaus Copernicus University in Torun, Poland

${ }^{3}$ Oncology Centre - Prof. Franciszek Łukaszczyk Memorial Hospital in Bydgoszcz, Bydgoszcz, Poland

${ }^{4}$ Chair and Department of Rehabilitation, Ludwik Rydygier Collegium Medicum in Bydgoszcz, Nicolaus Copernicus University in Torun, Poland

${ }^{5}$ Department of Tumour Pathology and Pathomorphology, Ludwik Rydygier Collegium Medicum in Bydgoszcz, Nicolaus Copernicus University in Torun, Poland

${ }^{6}$ Department of Cellular and Structural Biology, The University of Texas Health Science Center, San Antonio, TX, USA

${ }^{7}$ Department of Geriatric, South Texas Veterans Health System, The University of Texas Health Science Center, San Antonio, TX, USA
\end{abstract}

A 55-year-old man presented with periumbilical pain associated with the development of brownish discoloration near the umbilicus. The condition started 6 months before seeking medical attention. The pain increased particularly at the site of the brownish lesion and became more intense when the patient touched the site of the lesion. The gradual increase in pain severity as well as the noticeable changes of the skin lesion (the brownish discoloration became harder, larger and darker in hue) led the patient to seek medical advice. The patient has not been exposed to prominent risk factors being a non-smoker, since he works in a risk-free environment as a mechatronic engineer. Unfortunately, he has a positive family history of malignancies, which was unfavorable with his delayed diagnosis. His father died at the age of 72 due to lung cancer and his mother is a survivor of basal cell carcinoma of the nasomaxillary region.

Upon skin examination, the non-ulcerated skin lesion has been revealed. Melanoma malignum cutis has been identified after performing a preoperative dermatoscopy (Figure 1). The tumor was subsequently removed in one piece and the tissue identified histologically as a pT2a malignancy. The lesion has the following properties: dimensions of $2.3 \times 2 \times 1 \mathrm{~cm}$; Breslow thickness of $1.9 \mathrm{~mm}$; mitotic index of 4 mitoses $/ 5 \mathrm{HPF}$; margin of at least $0.1 \mathrm{~cm}$ healthy tissue; positive staining patterns of HMB-45 (+),
Melan A (+) and S100 (+) markers; and negative CK AE1/ AE3 (-) marker (Figure 2).

Following tumor resection, the patient was subjected to further investigation including abdominal and retroperitoneal ultrasound as well as pelvic ultrasound. The abdominal ultrasound showed hypo-echoic mass

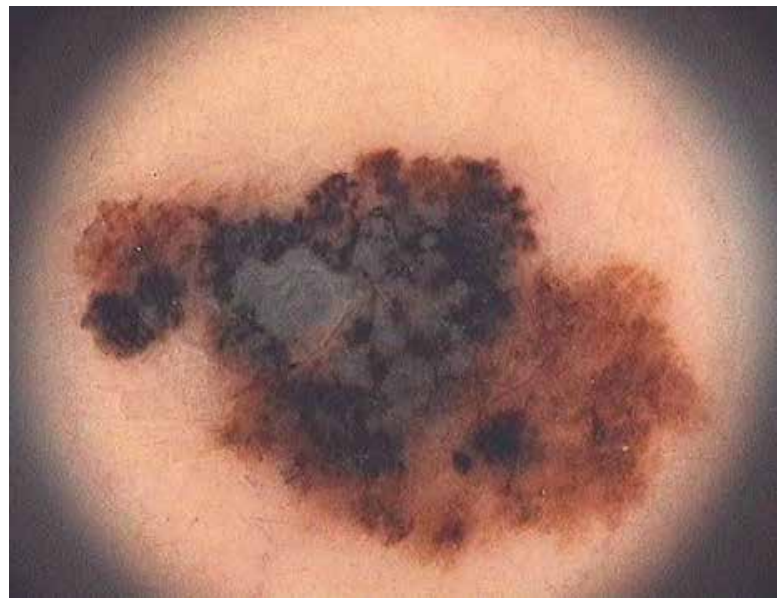

Figure 1. Surface microscopy using FotoFinder dermoscope dynamic $800 \mathrm{HD}$. All axes show asymmetric contours, irregular pigmentation, irregular dots and streaks. Additional blue whitish Weil and atypical pigment network were noted

Address for correspondence: MSc Maciej Nowacki MD, PhD, Chair and Department of Surgical Oncology, Ludwik Rydygier Collegium Medicum, Nicolaus Copernicus University, Oncology Centre - Prof. Franciszek Łukaszczyk Memorial Hospital, 2 Romanowskiej St, 85-796 Bydgoszcz, Poland, e-mail: maciej.s.nowacki@gmail.com Received: 4.12.2016, accepted: 5.12.2016. 

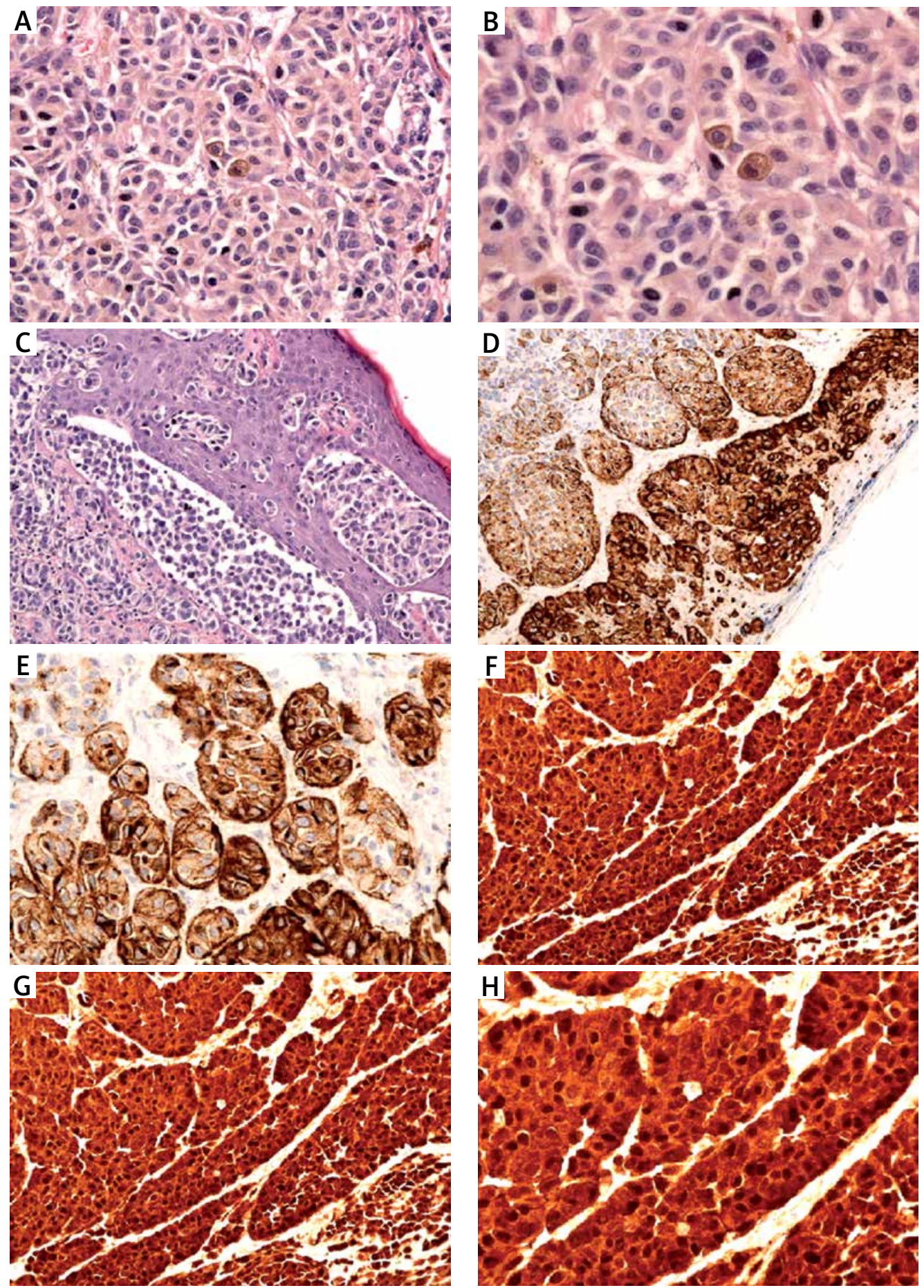

Figure 2. Histological staining of skin specimens show pT2a malignancy and Breslow thickness of $1.9 \mathrm{~mm}$. A - HE staining with magnification 400×, B - HE staining with magnification 600×, C - HE staining with magnification 200×, D - HMB45 staining with magnification 200×, E - HMB45 staining with magnification 400×, F-Melan-A staining with magnification 400x, G - S100 staining with magnification 200x, H - S100 staining with magnification 400x

with dimensions of $44 \times 26 \times 33 \mathrm{~mm}$ localized on the left umbilical region slightly lateral to the midline suggesting a pathological lymph node that needed further investigation. On the other hand, the retroperitoneal and pelvic scans were negative. More radical therapy was performed on the patient including an expanded resection but the tissue histopathology failed to show any evidence of the presence of cancer cells. 

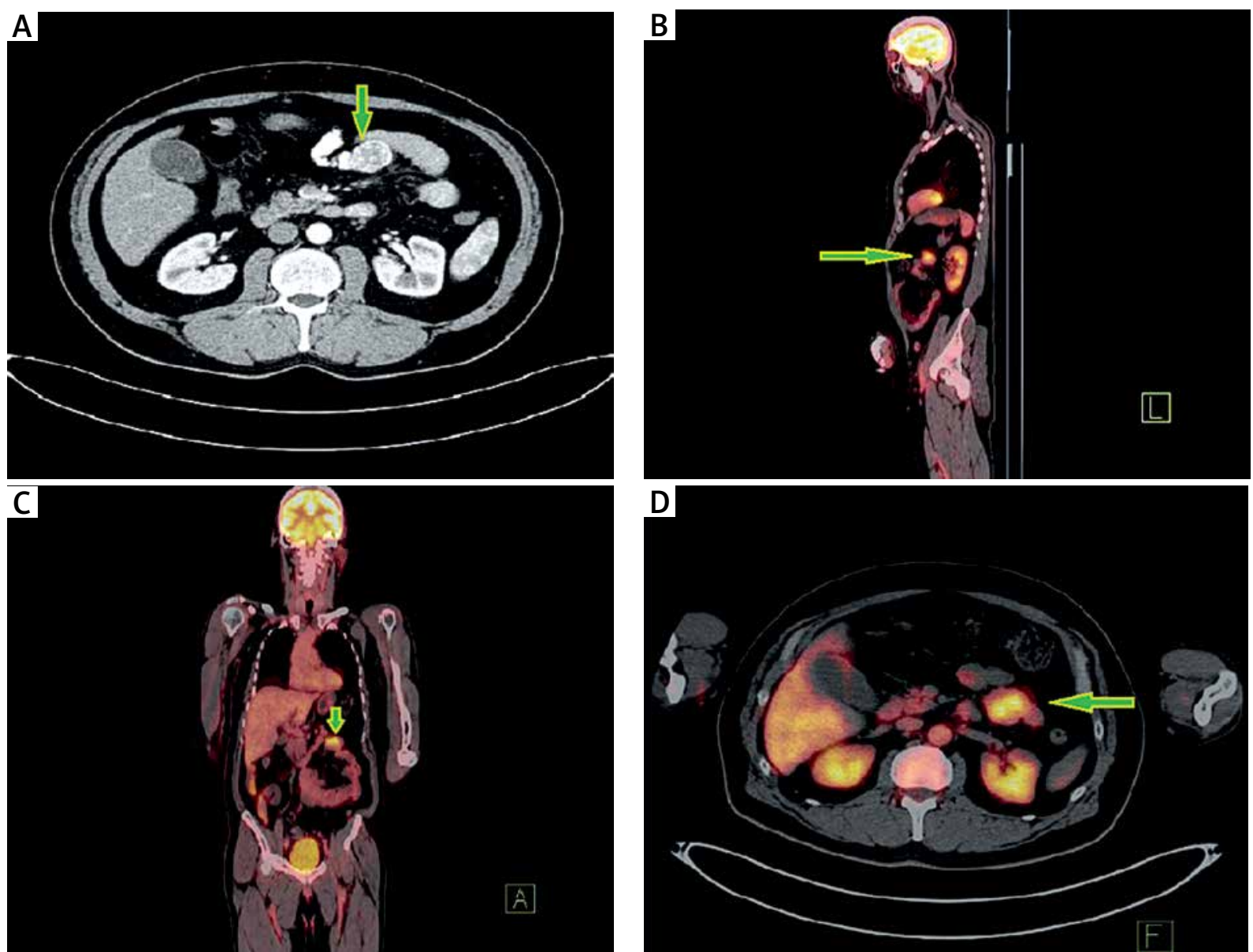

Figure 3. The CT and PET/CT scanning images. (A) Computed tomography, the arrow indicates a pathological mass $(37 \times 41 \mathrm{~mm})$ with inhomogeneous and hypoechogenic echotexture closely adhering to the small bowel loop. The primarily lesion was characterized as metastasis or gastrointestinal stromal tumor (GIST) and (B, C, D) by positron emission tomography. The arrow indicates tumor ( $33 \mathrm{~mm}$; SUV 6.1) localized in the mesentery beside the intestine loop in the left side of the middle region of the abdomen

Further findings were obtained after performing an abdominal CT scan. We found a pathological mass of $37 \times 41 \mathrm{~mm}$ with inhomogeneous and hypo-echogenic texture (Figure 3). Closely adhering to the small bowel loop, we found gastrointestinal stromal tumor (GIST). In order to ensure patient safety and due to the high morbidity and mortality of this metastatic tumor, the patient subsequently underwent a FUSION PET (PET/ $\mathrm{CT}$ ) scan using 18F-FDG imaging on the Biograph $\mathrm{mCT}$ 20. Basic parameters were measured including the blood glucose level, which showed $116 \mathrm{mg} / \mathrm{dl}$. The patient was injected with $500 \mathrm{MBq}$ of FDG and focal mass and the data showed that $12 \mathrm{~mm}$ of SUV 5.0 in the descending colon with a tumor size of $33 \mathrm{~mm}$. The SUV 6.1 is localized in the mesentery alongside of the intestinal loop on the left-hand side of the middle region of the abdomen (Figure 3).

After the PET/CT scan, the patient was referred for a colonoscopy that has been performed using the Olym- pus CF-Q180AL. The colonoscopy allowed the whole large intestine to be examined more clearly. The examination of ostium appendicis vermiformis and ileocecal valve became clearly visible. The data showed that the patient had an enlarged hemorrhoid while the intestinal wall and mucosa were normal. The Boston Bowel Preparation scale (BBPS) measured as $6 / 9$ points $(2+2+2)$ and no other pathological changes were observed. There were few doubts of the clinical diagnosis and the differentiation of the potential metastatic formation of malignant melanoma due to the lack of previous reports of similar cases. Next, the patient underwent exploratory laparoscopy and possible curative resection (Figure 4).

In the exploratory laparoscopy, $6 \mathrm{~cm}$ of the small intestine was found and resected. The surgical cut localizes $4.7 \mathrm{~cm}$ of pathological change and further $1.2 \mathrm{~cm}$ of nonpathological tissue. A final gross examination confirmed the presence of GIST. The characterization of the tumor is the following: tumor type pT2 with a low mitotic rate at 

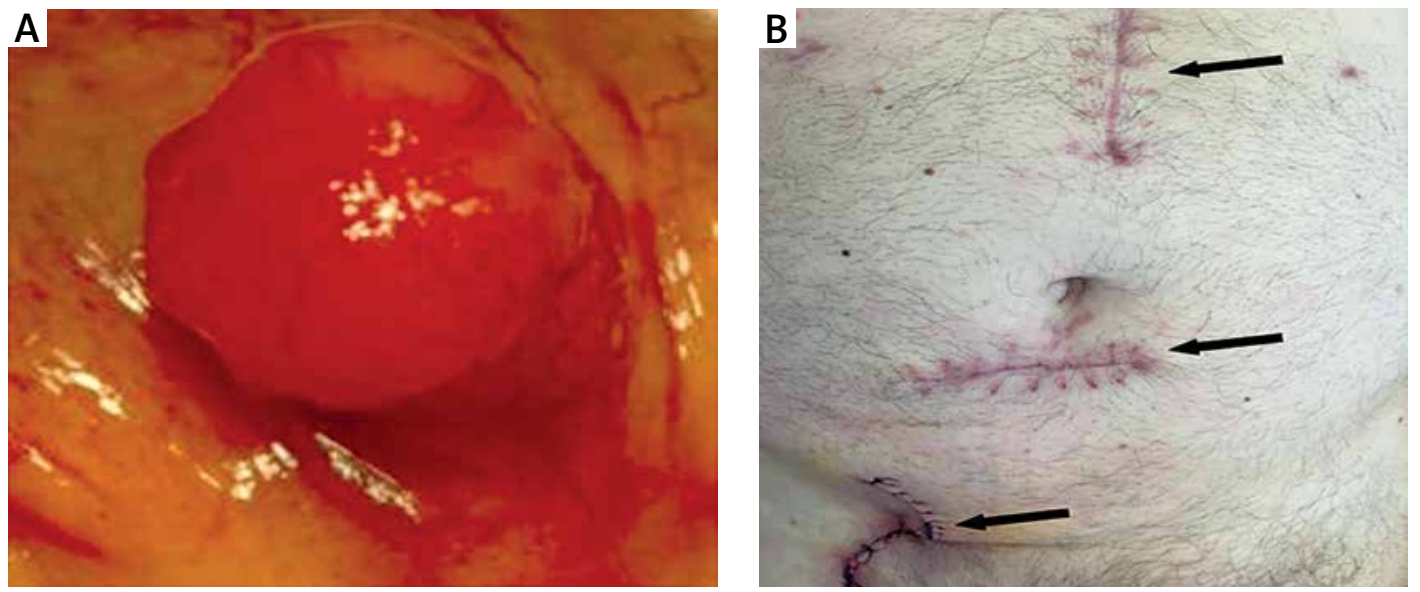

Figure 4. Intraoperative imaging showed the GIST tumor and postoperative abdominal scars presentation. A - Explorative laparoscopy showed tumor formation classified latterly as gastrointestinal stromal tumor (GIST). B - The arrows indicate postoperative scars after primary MM excision, laparoscopic GIST resection and lymph node dissection

stage IA of 1 mitose/50 HPF (Figure 5). In addition, the proliferation maker of Ki67 detected by immunohistochemical staining showed positive cells but did not exceed 5\%. Other markers showed some changes including CD34 (+), CD99 (+), CD117 (+), CK AE1/AE3 (-), Desmin (-), DOG-1 $(+)$, EMA (-), MA (-), Myogenin $(-)$, S100 (-), SMA (+) and Vimentin (+).

The lymphoscintigraphy and sentinel lymph node biopsy (SNB) were also performed. The SNB examination showed 2 nodes, the first node was $1.2 \mathrm{~cm}$ with lymph node metastasis and the second node was inflamed with a size of $0.5 \mathrm{~cm}$. Therefore, the patient underwent a wider excision of these lesions. Six months later, the patient was in good condition and he was also undergoing periodic observation as part of his postoperative follow-up.

Many studies showed the synchronous coincidence of double or multiple cancers [1, 2] and such occurrences are expected to be increased [3, 4]. Nowadays, there are only few detailed studies of synchronous cases of melanoma and other malignancies such as carcinomas of the large intestine, breast and prostate [5-7]. Up to now, there has been only one published case study that refers to synchronously diagnosed malignant melanoma (involving the right hard palate) and GIST [8]. In addition, there is one-center analysis indicating that multiple cancer occurrence may be considered as a coincidence but without details on the anatomical location or whether this case can be classified as metachronous or synchronous [9].

A well-known conclusion has been made that diagnostic imaging and other novel diagnosis modalities are important in providing early diagnosis which lead to early management and less complications to ensure catching many tumors at the early stage $[10,11]$. This is particularly true for globally synchronous GIST tumors, which are often detected incidentally during diagnostic processes or treatment [12]. Moreover, those patients are frequently diagnosed with an asynchronous occurrence of another malignancy during follow-up to their treatment [13]. Those double or multiple coincidences of GIST are mainly observed with other primary gastrointestinal malignancies [14].

However, individual cases related to other types of cancer are increasing since such data are available for comparative geographical, demographical and epidemiological analysis [15]. Such data analysis will be available for any research investigator in Poland that has only detected GIST as a secondary neoplasm while studies in other countries have observed it within the primary site of cancer [16].

The malignant melanoma is one of common types of skin cancer and can occur in many other organs. There is only a small number of case studies that referred to the synchronous coincidence between malignant melanoma and other neoplasms [5-17]. Such cases are characterized as very rare and incidental cases. Examples of these cases include cases of anorectal malignant melanoma coexisting with adenocarcinoma of the sigmoid colon and cases of double cancer incidences that involve esophageal MM and adenocarcinoma of the lung [18, 19].

Up to date, there has been only one published case study of synchronous double cancer involving melanoma malignum and GIST [8]. This case concerns a 78-yearold woman that differs from this case whereas there was no detection of pathological sentinel lymph nodes or any details of symptoms reported by the patient linked to GIST. However, there was a comparable absence of distant metastases and diagnostic track involving diagnostic imaging examination. Both cases have involved quick and early diagnosis as well as the relatively fast application of effective treatment.

So far no cases of occurrence of synchronous double cancer with both malignant periumbilical melanoma and GIST have been published. Therefore, reporting such 

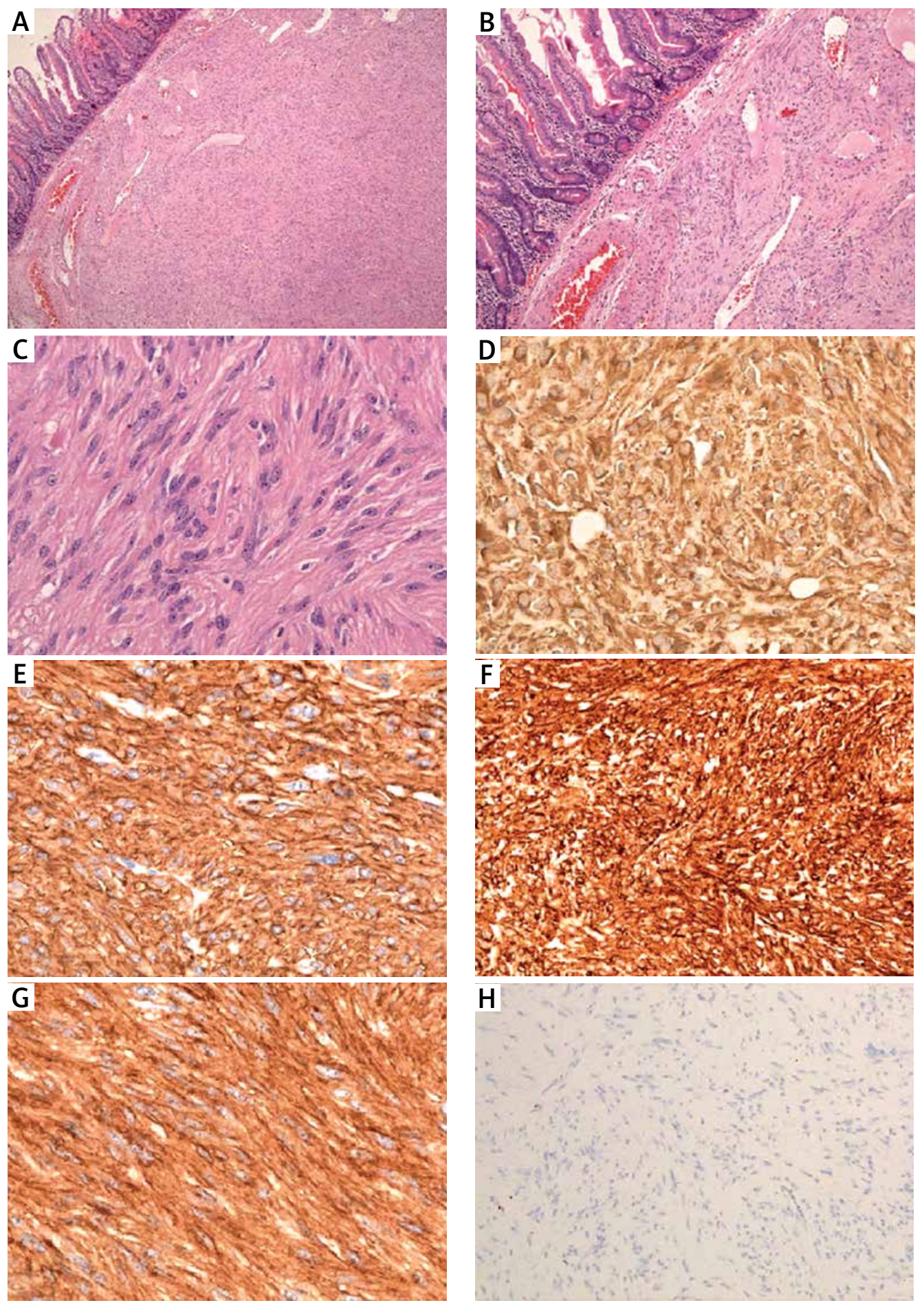

Figure 5. Histological examination of surgical skin specimens confirmed the presence of a gastrointestinal stromal tumor (GIST). The results confirmed the presence of GIST, type pT2 with a low mitotic rate at stage IA. A - HE staining - GIST with magnification 40x, B - HE staining - GIST with magnification 100×, C - HE staining - GIST with magnification 400×, D - VIM staining - GIST, with magnification 400x, E - DOG1 staining - GIST with magnification 400x, F-CD34 staining - GIST with magnification 200×, G - CD117 staining - GIST with magnification 400×, H - CKAE1-AE3 staining - GIST with magnification 200x 
cases in the future will increase our knowledge to avoid misdiagnosis and delayed therapy for the double cancer cases. Our report highlights some questions that might need further exploration of whether use of additional expand diagnostic protocols could help to avoid clinical omissions in rare cases and unexpected multiple cancer coincidences. In such rare cases, the right planning treatment will be a great benefit for those patients. The report provides a better guide for all cases of synchronous double or multiple cancers that are still growing with unexpected coincidences that are more likely to emerge.

\section{Conflict of interest}

The authors declare no conflict of interest.

\section{References}

1. Takayoshi K, Sagara K, Uchino K, et al. A case of metastatic renal cell carcinoma and bile duct carcinoma treated with a combination of sunitinib and gemcitabine. BMC Cancer 2015; 15: 426.

2. Song T, Seong SJ, Bae DS, et al. Prognostic factors in women with synchronous endometrial and ovarian cancers. Int J Gynecol Cancer 2014; 24: 520-7.

3. Li X, Lin S, Zhang Y, Wang H. Synchronous primary esophageal squamous cell carcinoma and gastric adenocarcinoma: analysis of 41 cases treated in a single institution. Sci Rep 2015; 5: 13335

4. Kamp K, Damhuis RA, Feelders RA, de Herder WW. Occurrence of second primary malignancies in patients with neuroendocrine tumors of the digestive tract and pancreas. Endocr Relat Cancer 2012; 19: 95-9.

5. Cooper CL, Murali R, Doubrovsky A, et al. Synchronous and metachronous malignancies in patients with melanoma: a clinicopathologic study highlighting the role of fine-needle biopsy cytology and potential diagnostic pitfalls. Melanoma Res 2010; 20: 203-11.

6. Betti R, Gualandri L, Vergani R, et al. Really synchronous cutaneous melanomas: serendipity or need for prevention? Eur J Dermatol 2009; 19: 258-9.

7. Kiedrowicz M, Halczak M, Kładny J, et al. Melanoma of unknown primary origin coexisting with early-onset multifocal basal cell carcinoma. Postep Derm Alergol 2015; 32: 320-2.

8. Nagai K, Matsumura Y, Nomura J, et al. A case of double cancer involving oral malignant melanoma and gastrointestinal stromal tumor (GIST). Int J Oral Maxillofac Surg 2005; 34 328-30.

9. Agaimy A, Wünsch PH, Sobin LH, et al. Occurrence of other malignancies in patients with gastrointestinal stromal tumors. Semin Diagn Pathol 2006; 23: 120-9.

10. Chen SH, Chan SC, Chao YK, Yen TC. Detection of synchronous cancers by fluorodeoxyglucose positron emission tomography/computed tomography during primary staging workup for esophageal squamous cell carcinoma in Taiwan. PLoS One 2013; 8: e82812.

11. Sturludóttir M, Martling A, Carlsson S, Blomqvist L. Synchronous rectal and prostate cancer: the impact of MRI on incidence and imaging findings. Eur J Radiol 2015; 84: 563-7.

12. Joensuu H. Gastrointestinal stromal tumor (GIST). Ann Oncol 2006; 17 (Suppl 10): x280-6.
13. Wada Y, Koizumi T, Yokoyama T, et al. Synchronous gastrointestinal stromal tumor and primary lung adenocarcinoma. Intern Med 2012; 51: 2407-10.

14. Kaur R, Bhalla S, Nundy S, Jain S. Synchronous gastric gastrointestinal stromal tumor (GIST) and other primary neoplasms of gastrointestinal tract: report of two cases. Ann Gastroenterol 2013; 26: 356-9.

15. Lin M, Lin JX, Huang CM, et al. Prognostic analysis of gastric gastrointestinal stromal tumor with synchronous gastric cancer. World J Surg Oncol 2014; 12: 25.

16. Wronski M, Ziarkiewicz-Wroblewska B, Gornicka B, et al. Synchronous occurrence of gastrointestinal stromal tumors and other primary gastrointestinal neoplasms. World J Gastroenterol 2006; 12: 5360-2.

17. Yagi KI, Wani HU, Tinguria MB, Al-Qahtani DM. Malignant melanoma of the skin: a case report from the eastern region (Dammam Central Hospital), Saudi Arabia and epidemiological review. J Family Comm Med 2000; 7: 67-71.

18. Teke Z, Ozogul YB, Aydog G, et al. Multiple synchronous anorectal malignant melanoma coexisting with adenocarcinoma of the sigmoid colon. Indian J Surg 2013; 75: 164-6.

19. Yano M, Shiozaki H, Murata A, et al. Primary malignant melanoma of the esophagus associated with adenocarcinoma of the lung. Surg Today 1998; 28: 405-8. 